Pacific

Journal of

Mathematics

A CONSTRUCTION OF LOGARITHMIC 1-FORMS ON HYPERPLANE ARRANGEMENTS

ScOtт K. Imig 


\title{
A CONSTRUCTION OF LOGARITHMIC 1-FORMS ON HYPERPLANE ARRANGEMENTS
}

\author{
ScotT K. Imig
}

\begin{abstract}
We realize an idea suggested in the literature by Yuzvinsky, giving a construction of logarithmic 1-forms from syzygies of linear functionals.
\end{abstract}

\section{Introduction.}

Let $V$ be a vector space of finite dimension $l$ over a field $\mathbf{K}$. An arrangement is a finite set $\mathcal{A}=\left\{X_{1}, \ldots, X_{n}\right\}$ of hyperplanes in $V$. We assume the arrangement is central, i.e., all hyperplanes contain the origin. Let $S$ be the symmetric algebra of $V^{*}$, which is canonically isomorphic to the polynomial algebra $\mathbf{K}\left[x_{1}, \ldots, x_{l}\right]$ for any basis $\left(x_{1}, \ldots, x_{l}\right)$ of $V^{*}$. For each $X \in \mathcal{A}$, fix a linear functional $\alpha_{X} \in V^{*}$ with $\operatorname{ker} \alpha_{X}=X$. Let $Q=\prod_{X \in \mathcal{A}} \alpha_{X}$.

The module of logarithmic differential forms with poles along a divisor was introduced by Deligne [De] for a divisor with normal crossings, and the definition was generalized by Saito [Sa] to an arbitrary divisor. Extensive research has been done into the structure of this module when the divisor is a union of hyperplanes, but some important motivating questions, such as [OT, Conjecture 4.138], remain unanswered.

Let $\Omega^{q}[V]$ represent the $S$-module of differential $q$-forms on $V$ with coefficients from $S$ and let

$$
\Omega^{q}(\mathcal{A})=\left\{\begin{array}{l|l}
\omega & \begin{array}{l}
\omega \text { is a global rational differential } q \text {-form on } V \\
\text { such that } Q \omega \in \Omega^{q}[V] \text { and } Q d \omega \in \Omega^{q+1}[V]
\end{array}
\end{array}\right\} .
$$

This is the module of logarithmic differential q-forms corresponding to $\mathcal{A}$. The $S$-module $\Omega^{1}(\mathcal{A})$ and its dual are important algebraic objects studied in arrangement theory, and we herein restrict our attention to the case $q=1$.

The present work is motivated by several discoveries by Rose, Terao, Yuzvinsky, and Ziegler, appearing in $[\mathbf{R T}],[\mathbf{Y u}]$, and $[\mathbf{Z i}]$, which suggest that there may be a construction of generators for $\Omega^{1}(\mathcal{A})$ from either combinatoric data or simple algebraic information about the functionals $\alpha_{X}$. Some of these results can be elegantly formulated by using a chain complex of relation spaces, here denoted $\bigoplus R .\left(\mathcal{A}_{X}\right)$, which has appeared in the papers $[\mathbf{B T}]$ by Brandt and Terao and $[\mathbf{Y u}]$ by Yuzvinsky. The results in $[\mathbf{R T}]$ and $[\mathbf{Z i}]$ imply that if the second relation space, $\bigoplus R_{2}\left(\mathcal{A}_{X}\right)$, vanishes, then the logarithmic forms $d \alpha_{X} / \alpha_{X}$ generate the whole $S$-module $\Omega^{1}(\mathcal{A})$. In [Yu], Yuzvinsky 
gives forms which generate the module $\Omega^{1}(\mathcal{A})$ whenever the third relation space, $\bigoplus R_{3}\left(\mathcal{A}_{X}\right)$, vanishes.

In $[\mathbf{Y u}$ ], Yuzvinsky suggests that there may be an interesting construction of logarithmic 1-forms from elements of $\bigoplus R$. $\left(\mathcal{A}_{X}\right)$. In this paper we realize Yuzvinsky's idea, giving an explicit construction. The proof that the resulting forms are logarithmic proceeds by the method of deletion and restriction, which was introduced by Zaslavsky in $[\mathbf{Z a}]$ and has become very pervasive in the algebraic theory of arrangements. One family of arrangements for which $\Omega^{1}(\mathcal{A})$ is generated by these 1 -forms is the family associated to the real reflection groups of type $A$.

In Section 2, we define the chain complex $\bigoplus R$. $\left(\mathcal{A}_{X}\right)$. In Sections 3 and 4, we apply Zaslavsky's method of deletion and restriction to $\bigoplus R$. $\left(\mathcal{A}_{X}\right)$. In Sections 5 and 6 , we recursively construct logarithmic 1-forms $\omega_{\mu}$ associated to elements $\mu$ of $R_{k}\left(\mathcal{A}_{X}\right)$ and prove some properties of these forms. In Section 7 , we show that $\Omega^{1}(\mathcal{A})$ is generated by these forms for reflection arrangements of type $A$.

\section{A chain complex of relation spaces.}

This section introduces a chain complex, denoted $\bigoplus R .\left(\mathcal{A}_{X}\right)$, which has appeared in $[\mathbf{B T}]$ and in $[\mathbf{Y u}]$.

Let $L$ be the poset of all intersections of the hyperplanes of $\mathcal{A}$, ordered by reverse inclusion, with $V$ itself regarded as the empty intersection. It is well-known that $L$ is a geometric lattice. We let $L_{k}$ represent the lattice elements of codimension $k$. For any $Y \in L$, let $\mathcal{A}_{Y}$ denote the subset of $\mathcal{A}$ consisting of all $X \in \mathcal{A}$ with $Y \subset X$. Note that $\mathcal{A}=L_{1} \subset L$.

Let $R_{1}(\mathcal{A})$ be the vector space with basis $\left(e_{1}, \ldots, e_{n}\right)$ in one to one correspondence with the hyperplanes of $\mathcal{A}$. Note that there is an obvious isomorphism

$$
\bigoplus_{X \in L_{1}} R_{1}\left(\mathcal{A}_{X}\right) \longrightarrow R_{1}(\mathcal{A})
$$

so we may write $\bigoplus_{X \in L_{1}} R_{1}\left(\mathcal{A}_{X}\right)$ in place of $R_{1}(\mathcal{A})$. We will use this notation, since it is more convenient for the recursive definitions in later sections. Let $\delta_{1}^{\mathcal{A}}: \bigoplus_{X \in L_{1}} R_{1}\left(\mathcal{A}_{X}\right) \longrightarrow V^{*}$ be the linear map defined via $\delta_{1}^{\mathcal{A}} e_{i}=\alpha_{X_{i}}$. Let $R_{2}(\mathcal{A})=\operatorname{ker} \delta_{1}^{\mathcal{A}}$.

For $2 \leq k$, assume that we have vector spaces $R_{k}(\mathcal{A})$ and $R_{k-1}(\mathcal{A})$, depending on $\mathcal{A}$, such that $R_{k}(\mathcal{A}) \subset \bigoplus_{X \in L_{k-1}} R_{k-1}\left(\mathcal{A}_{X}\right)$. We define a new vector space $R_{k+1}(\mathcal{A})$.

Let $\delta_{k}^{\mathcal{A}}$ be the map

$$
\delta_{k}^{\mathcal{A}}: \bigoplus_{Y \in L_{k}} R_{k}\left(\mathcal{A}_{Y}\right) \longrightarrow \bigoplus_{X \in L_{k-1}} R_{k-1}\left(\mathcal{A}_{X}\right)
$$


induced by the canonical inclusions

$$
R_{k}\left(\mathcal{A}_{Y}\right) \hookrightarrow \bigoplus_{X \in L_{k-1}} R_{k-1}\left(\mathcal{A}_{X}\right)
$$

Let $R_{k+1}(\mathcal{A})=\operatorname{ker} \delta_{k}^{\mathcal{A}}$.

For $k>l$, note that $R_{k}(\mathcal{A})=0$. For simplicity, we will write $\delta_{i}^{\mathcal{A}}$ as $\delta_{i}$ for every $i$. For any $Y \in L$, write $\delta_{i}^{\mathcal{A}_{Y}}$ as $\delta_{i}^{Y}$ for every $i$. For any $k \geq 2, Y \in L_{k}$ and any $\mu \in \bigoplus_{Y \in L_{k}} R_{k}\left(\mathcal{A}_{Y}\right)$, let $\mu_{Y}$ denote the $Y$ coordinate of $\mu$. For any $Y \in L_{k}$ and $X \in L_{k-1}^{Y}$, observe that this defines the notation $\left(\mu_{Y}\right)_{X}$. It is convenient to extend this definition to all $X \in L_{k-1}$ by letting $\left(\mu_{Y}\right)_{X}=0$ in case $X \in L_{k-1} \backslash L_{k-1}^{Y}$. Notice that for $k \geq 2, X \in L_{k-1}$, the $X$ coordinate of $\delta_{k} \mu$ is $\sum_{Y \in L_{k}}\left(\mu_{Y}\right)_{X}$.

Observe that the sequence

$$
0 \longrightarrow R_{l}(\mathcal{A}) \stackrel{\delta_{l}}{\longrightarrow} \bigoplus_{X \in L_{l-1}} R_{l-1}\left(\mathcal{A}_{X}\right) \stackrel{\delta_{l-1}}{\longrightarrow} \cdots \stackrel{\delta_{2}}{\longrightarrow} \bigoplus_{X \in L_{1}} R_{1}\left(\mathcal{A}_{X}\right) \stackrel{\delta_{1}}{\longrightarrow} V^{*} \longrightarrow 0
$$

is a chain complex.

\section{Deletion and Restriction.}

We now apply Zaslavsky's method of deletion and restriction to the chain complex $\oplus R .\left(\mathcal{A}_{X}\right)$. For convenience in this section and later sections, we put

$$
V\left(p_{1}, \ldots, p_{m}\right)=\cap_{i=1}^{m} \operatorname{ker} p_{i}
$$

for any $p_{1}, \ldots, p_{m} \in V^{*}$.

Let $\theta \in V^{*}$. Let $\mathcal{A}^{\prime}=\mathcal{A} \backslash\{V(\theta)\}$. In particular, if $V(\theta) \notin \mathcal{A}$, then $\mathcal{A}^{\prime}=\mathcal{A}$. Let $\mathcal{A}^{\prime \prime}=\left\{X \cap V(\theta) \mid X \in \mathcal{A}^{\prime}\right\}$. For each $Z \in \mathcal{A}^{\prime \prime}$, choose some $\eta_{Z} \in V(\theta)^{*}$ with $\operatorname{ker} \eta_{Z}=Z$.

Again, we simplify notation. Let $L^{\prime \prime}$ denote $L\left(\mathcal{A}^{\prime \prime}\right)$ and for any $X \in L$, let $X^{\prime \prime}$ denote $X \cap V(\theta) \in L^{\prime \prime}$. For any $\alpha \in V^{*}$, let $\alpha^{\prime \prime}$ denote $\left.\alpha\right|_{V(\theta)}$. For $i \geq 1$, let $\delta_{i}^{\prime \prime}$ denote $\delta_{i}^{\mathcal{A}^{\prime \prime}}$.

Let $h_{0}^{\mathcal{A}}: V^{*} \longrightarrow V(\theta)^{*}$ be given by $h_{0}^{\mathcal{A}}(\alpha)=\alpha^{\prime \prime}$.

For any $X \in \mathcal{A}, X \neq V(\theta)$, notice that $\alpha_{X}^{\prime \prime}$ and $\eta_{X^{\prime \prime}}$ are $\mathbf{K}$-proportional. Thus we may let $\frac{\alpha_{X}^{\prime \prime}}{\eta_{X^{\prime \prime}}}$ denote the scalar $a \in \mathbf{K}$ such that $\alpha_{X}^{\prime \prime}=a \eta_{X^{\prime \prime}}$. Now define

$$
h_{1}^{\mathcal{A}}: \bigoplus_{X \in L_{1}} R_{1}\left(\mathcal{A}_{X}\right) \longrightarrow \bigoplus_{Z \in L_{1}^{\prime \prime}} R_{1}\left(\mathcal{A}_{Z}^{\prime \prime}\right)
$$

via

$$
e_{X} \mapsto \begin{cases}\frac{\alpha_{X}^{\prime \prime}}{\eta_{X^{\prime \prime}}} e_{X^{\prime \prime}} & \text { if } X \neq V(\theta), \text { and } \\ 0 & \text { if } X=V(\theta) .\end{cases}
$$


By definition of $h_{1}^{\mathcal{A}}$, the following diagram commutes.

$$
\begin{aligned}
& \bigoplus_{X \in L_{1}} R_{1}\left(\mathcal{A}_{X}\right) \stackrel{\delta_{1}}{\longrightarrow} V^{*} \\
& h_{1}^{\mathcal{A}} \downarrow \\
& \bigoplus_{Z \in L_{1}^{\prime \prime}} R_{1}\left(\mathcal{A}_{Z}^{\prime \prime}\right) \stackrel{\delta_{1}^{\prime \prime}}{\longrightarrow} V(\theta)^{*}
\end{aligned}
$$

Lemma 3.1. For any $Y \in L_{2}, h_{1}^{\mathcal{A}_{Y}} R_{2}\left(\mathcal{A}_{Y}\right) \subset R_{2}\left(\mathcal{A}_{Y^{\prime \prime}}^{\prime \prime}\right)$.

Proof. Let $\mu \in R_{2}\left(\mathcal{A}_{Y}\right)$. Observe that $\left(\mathcal{A}_{Y}\right)^{\prime \prime} \subset \mathcal{A}_{Y^{\prime \prime}}^{\prime \prime}$. By $(2), \delta_{1}^{\mathcal{A}_{Y}^{\prime \prime}} h_{1}^{\mathcal{A}_{Y}} \mu=$ $h_{0}^{\mathcal{A}_{Y}} \delta_{1}^{Y} \mu=h_{0}^{\mathcal{A}_{Y}} 0=0$.

\section{The chain map.}

We now recursively generalize the preceding construction and properties, obtaining a chain map. Let $k \geq 1$, and assume that we have a linear map

$$
h_{k}^{\mathcal{A}}: \bigoplus_{X \in L_{k}} R_{k}\left(\mathcal{A}_{X}\right) \longrightarrow \bigoplus_{Z \in L_{k}^{\prime \prime}} R_{k}\left(\mathcal{A}_{Z}^{\prime \prime}\right)
$$

so that, for any $Y \in L_{k}$,

$$
h_{k}^{\mathcal{A}_{Y}} R_{k+1}\left(\mathcal{A}_{Y}\right) \subset R_{k+1}\left(\mathcal{A}_{Y^{\prime \prime}}^{\prime \prime}\right) .
$$

When $k=1$, the assumption (3) amounts to Lemma 3.1. For the case $k>1$, the reader is referred to Lemma 4.1. We now define a new linear map

$$
h_{k+1}^{\mathcal{A}}: \bigoplus_{Y \in L_{k+1}} R_{k+1}\left(\mathcal{A}_{Y}\right) \longrightarrow \bigoplus_{W \in L_{k+1}^{\prime \prime}} R_{k+1}\left(\mathcal{A}_{W}^{\prime \prime}\right) .
$$

The construction proceeds coordinate by coordinate. For every $Y \in L_{k+1}$, assumption (3) gives rise to a composition

$$
R_{k+1}\left(\mathcal{A}_{Y}\right) \stackrel{h_{k}^{\mathcal{A}_{Y}}}{\longrightarrow} R_{k+1}\left(\mathcal{A}_{Y^{\prime \prime}}^{\prime \prime}\right) \hookrightarrow \bigoplus_{W \in L_{k+1}^{\prime \prime}} R_{k+1}\left(\mathcal{A}_{W}^{\prime \prime}\right) .
$$

If $\theta \notin Y^{\perp}$, then the inclusion in the composition (4) is the canonical injection. If $\theta \in Y^{\perp}$, then

$$
R_{k+1}\left(\mathcal{A}_{Y^{\prime \prime}}^{\prime \prime}\right)=R_{k+1}\left(\mathcal{A}_{Y}^{\prime \prime}\right)=0
$$

so the inclusion in composition (4) is the trivial inclusion $0 \hookrightarrow \bigoplus_{W \in L_{k+1}^{\prime \prime}}$ $R_{k+1}\left(\mathcal{A}_{W}^{\prime \prime}\right)$. The compositions (4) induce the linear map

$$
h_{k+1}^{\mathcal{A}}: \bigoplus_{Y \in L_{k+1}} R_{k+1}\left(\mathcal{A}_{Y}\right) \rightarrow \bigoplus_{W \in L_{k+1}^{\prime \prime}} R_{k+1}\left(\mathcal{A}_{W}^{\prime \prime}\right) .
$$


Note that we have, for any $W \in L_{k+1}^{\prime \prime}$,

$$
\left(h_{k+1}^{\mathcal{A}} \mu\right)_{W}=\sum_{\substack{Y \in L_{k+1} \\ Y^{\prime \prime}=W}} h_{k}^{\mathcal{A}_{Y}} \mu_{Y} .
$$

By definition of $h_{k+1}^{\mathcal{A}}$, the following diagram commutes.

$$
\begin{array}{cc}
\bigoplus_{Y \in L_{k+1}} R_{k+1}\left(\mathcal{A}_{Y}\right) \stackrel{\delta_{k+1}}{\longrightarrow} \bigoplus_{X \in L_{k}} R_{k}\left(\mathcal{A}_{X}\right) \\
\bigoplus_{k+1}^{\mathcal{A}} \downarrow & \downarrow_{k}^{\mathcal{A}} \\
\bigoplus_{W \in L_{k+1}^{\prime \prime}} R_{k+1}\left(\mathcal{A}_{W}^{\prime \prime}\right) \stackrel{\delta_{k+1}^{\prime \prime}}{\longrightarrow} & \bigoplus_{Z \in L_{k}^{\prime \prime}} R_{k}\left(\mathcal{A}_{Z}^{\prime \prime}\right)
\end{array}
$$

The proof of the following lemma is exactly analogous to Lemma 3.1.

Lemma 4.1. For any $Y \in L_{k}, h_{k+1} R_{k+1}\left(\mathcal{A}_{Y}\right) \subset R_{k+1}\left(\mathcal{A}_{Y^{\prime \prime}}^{\prime \prime}\right)$.

For simplicity, we will write $h_{i}^{\mathcal{A}}$ as $h_{i}$ for every $i$. In general, the maps $h_{i}^{\mathcal{A}_{X}}$ with $X \in L$ will be written $h_{i}^{X}$. We have now shown that the following diagram commutes.

$$
\begin{aligned}
& \cdots \longrightarrow \bigoplus_{X \in L_{k}} R_{k}\left(\mathcal{A}_{X}\right) \stackrel{\delta_{k}}{\longrightarrow} \bigoplus_{X \in L_{k-1}} R_{k-1}\left(\mathcal{A}_{X}\right) \stackrel{\delta_{k-1}}{\longrightarrow} \\
& h_{k} \downarrow \quad h_{k-1} \downarrow \\
& \cdots \longrightarrow \bigoplus_{Z \in L_{k}^{\prime \prime}} R_{k}\left(\mathcal{A}_{Z}^{\prime \prime}\right) \stackrel{\delta_{k}^{\prime \prime}}{\longrightarrow} \bigoplus_{Z \in L_{k-1}^{\prime \prime}} R_{k-1}\left(\mathcal{A}_{Z}^{\prime \prime}\right) \stackrel{\delta_{k-1}^{\prime \prime}}{\longrightarrow} \\
& \cdots \stackrel{\delta_{2}}{\longrightarrow} \bigoplus_{X \in L_{1}} R_{1}\left(\mathcal{A}_{X}\right) \stackrel{\delta_{1}}{\longrightarrow} V^{*} \stackrel{\delta_{0}}{\longrightarrow} 0 \\
& h_{1} \downarrow \quad h_{0} \downarrow \\
& \cdots \stackrel{\delta_{2}^{\prime \prime}}{\longrightarrow} \bigoplus_{Z \in L_{1}^{\prime \prime}} R_{1}\left(\mathcal{A}_{Z}^{\prime \prime}\right) \stackrel{\delta_{1}^{\prime \prime}}{\longrightarrow} V(\theta)^{*} \stackrel{\delta_{0}^{\prime \prime}}{\longrightarrow} 0 .
\end{aligned}
$$

The commutativity of the big diagram is summarized in the following proposition.

Proposition 4.2. Let $k \geq 1$. Let $\mu \in \bigoplus_{X \in L_{k}} R_{k}\left(\mathcal{A}_{X}\right)$. Then

$$
\delta_{k}^{\prime \prime} h_{k} \mu=h_{k-1} \delta_{k} \mu .
$$

The following observation is useful in the proof of our main result, Theorem 6.5.

Lemma 4.3. Let $2<k \leq l, Y \in L_{k}$, and $\mu \in R_{k}\left(\mathcal{A}_{Y}\right)$. Assume $\theta \in Y^{\perp}$. Then

$$
\sum_{\substack{X \in L_{k-1}^{Y} \\ \theta \notin X^{\perp}}} h_{k-2}^{X} \mu_{X}=0 .
$$


Proof. Note that the codomain of $h_{k}^{Y}: R_{k}\left(\mathcal{A}_{Y}\right) \longrightarrow R_{k}\left(\left(\mathcal{A}_{Y}\right)^{\prime \prime}\right)$ is 0 , and so $h_{k}^{Y}$ is the zero map. Thus $\delta_{k}^{\left(\mathcal{A}_{Y}\right)^{\prime \prime}} h_{k}^{Y} \mu=\delta_{k}^{\left(\mathcal{A}_{Y}\right)^{\prime \prime}} 0=0$. By Proposition 4.2, this implies $h_{k-1}^{Y} \delta_{k}^{Y} \mu=0$. The map $\delta_{k}^{Y}$ is the inclusion $R_{k}\left(\mathcal{A}_{Y}\right) \hookrightarrow$ $\bigoplus_{X \in L_{k-1}} R_{k-1}\left(\mathcal{A}_{X}\right)$, so $h_{k-1}^{Y} \mu=h_{k-1} \delta_{k}^{Y} \mu=0$. Now observe $L_{k-1}\left(\left(\mathcal{A}_{Y}\right)^{\prime \prime}\right)=$ $\{Y\}$. Also observe, for $X \in L_{k-1}^{Y}, X^{\prime \prime}=Y$ if and only if $\theta \notin X^{\perp}$. Therefore, by $(5)$,

$$
h_{k-1}^{Y} \mu=\sum_{\substack{X \in L_{k-1}^{Y} \\ \theta \notin X^{\perp}}} h_{k-2}^{X} \mu_{X} .
$$

This completes the proof.

\section{Construction of differential 1-forms.}

In this section we construct differential 1-forms $\omega_{\mu}$ in a recursive manner. It will be shown in the next section that these forms are logarithmic.

Lemma 5.1. Let $Y \in L_{k}, 1 \leq j \leq k$, and $X \in L_{k-j}^{Y}$. Let $\left(\beta_{1}, \ldots, \beta_{k}\right)$ be any basis of $Y^{\perp}$. Let $\left\langle\beta_{1}, \ldots, \beta_{j}\right\rangle$ denote the span of $\left\{\beta_{1}, \ldots, \beta_{j}\right\}$ in $Y^{\perp}$. If $\left\langle\beta_{1}, \ldots, \beta_{j}\right\rangle \cap X^{\perp}=0$, then:

(i) There is a unique basis $C^{X}=\left(\gamma_{j+1}^{X}, \ldots, \gamma_{k}^{X}\right)$ of $X^{\perp}$ so that

$$
\left.C^{X}\right|_{V\left(\beta_{1}, \ldots, \beta_{j}\right)}=\left.\left(\beta_{j+1}, \ldots, \beta_{k}\right)\right|_{V\left(\beta_{1}, \ldots, \beta_{j}\right)} .
$$

(ii) Let $j+1 \leq i \leq k$. Let any $\eta_{i} \in X^{\perp}$ have the property $\left.\eta_{i}\right|_{V\left(\beta_{1}, \ldots, \beta_{j}\right)}=$ $\left.\beta_{i}\right|_{V\left(\beta_{1}, \ldots, \beta_{j}\right)}$. Then $\eta_{i}=\gamma_{i}^{X}$.

Proof. Note that $Y^{\perp}$ has the internal direct sum decomposition

$$
Y^{\perp}=\left\langle\beta_{1}, \ldots, \beta_{j}\right\rangle \oplus X^{\perp} .
$$

Let $\pi_{1}: Y^{\perp} \longrightarrow\left\langle\beta_{1}, \ldots, \beta_{j}\right\rangle$ and $\pi_{2}: Y^{\perp} \longrightarrow X^{\perp}$ be the projections. For each $i, j+1 \leq i \leq k$, let $\gamma_{i}^{X}=\pi_{2}\left(\beta_{i}\right)$, and let $C^{X}=\left(\gamma_{j+1}^{X}, \ldots, \gamma_{k}^{X}\right)$. This proves existence in (i). Part (ii) now follows. The uniqueness of $C^{X}$ in (i) is a direct consequence of (ii).

For $Y \in L_{k}(\mathcal{A}), X \in L_{k-1}^{Y}$, let $\left(\beta_{1}, \ldots, \beta_{k}\right)$ be a basis of $Y^{\perp}$ with $\beta_{1} \notin X^{\perp}$. Since $Y^{\perp} / X^{\perp}$ is one-dimensional and $\beta_{1}^{X} \not \equiv 0 \bmod X^{\perp}$, there is a scalar $a_{i}^{X} \in \mathbf{K}$ (possibly zero) so that

$$
\beta_{i}^{X} \equiv a_{i}^{X} \beta_{1}^{X} \bmod X^{\perp} .
$$

Lemma 5.2. Let $Y \in L_{k}(\mathcal{A})$ and $X \in L_{k-1}^{Y}$. Let $\left(\beta_{1}, \ldots, \beta_{k}\right)$ be a basis of $Y^{\perp}$ with $\beta_{1} \notin X^{\perp}$, and $C^{X}=\left(\gamma_{2}^{X}, \ldots, \gamma_{k}^{X}\right)$ be as in Lemma 5.1. Then for every $i, 2 \leq i \leq k, \gamma_{i}^{X}=\beta_{i}-a_{i}^{X} \beta_{1}$.

Proof. Note that $\left.\left(\beta_{i}-a_{i}^{X} \beta_{1}\right)\right|_{V\left(\beta_{1}\right)}=\left.\beta_{i}\right|_{V\left(\beta_{1}\right)}$, and $\beta_{i}-a_{i}^{X} \beta_{1} \in X^{\perp}$. The result follows by Lemma 5.1(ii). 
Let $1 \leq k \leq l, Y \in L_{k}$, and $\mu \in R_{k}\left(A_{Y}\right)$ be fixed. Let $B=\left(\beta_{1}, \ldots, \beta_{k}\right)$ be a $k$-tuple of elements in $Y^{\perp}$. If $B$ is linearly dependent or $\mu=0$, then we define $\omega_{\mu}(B)=0$. In particular, if $B=(0, \ldots, 0)$, then $\omega_{\mu}(B)=0$. Otherwise, $\omega_{\mu}(B)$ is defined recursively as follows.

Suppose $k=1$. Then $Y \in L_{1}=\mathcal{A}$, and, since $\mu \in R_{1}\left(\mathcal{A}_{Y}\right), \mu$ is of the form $c e_{Y}$ for some $c \in \mathbf{K}$. Furthermore, $B=\left(\beta_{1}\right)$ is a 1-tuple. We let $\omega_{\mu}(B)=\frac{c}{\beta_{1}} d \alpha_{Y}$.

Now suppose $k \geq 2$. Assume for any $X \in L_{k-1}$, any $(k-1)$-tuple $C$ of elements of $X^{\perp}$, and any $\nu \in R_{k-1}\left(\mathcal{A}_{X}\right)$, we have a global rational 1-form $\omega_{\nu}(C)$ on $V$.

Observe that if $X \in L_{k-1}^{Y}$ and $\beta_{1} \notin X^{\perp}$, then Lemma 5.1 gives us a basis $C^{X}=\left(\gamma_{2}^{X}, \ldots, \gamma_{k}^{X}\right)$ of $X^{\perp}$ so that $\left.C^{X}\right|_{V\left(\beta_{1}\right)}=\left.\left(\beta_{2}, \ldots, \beta_{k}\right)\right|_{V\left(\beta_{1}\right)}$. We may extend the definition of $C^{X}$ to any $X \in L_{k-1}^{Y}$ via

$$
C^{X}= \begin{cases}\left(\gamma_{2}^{X}, \ldots, \gamma_{k}^{X}\right) & \text { if } \beta_{1} \notin X^{\perp} \\ (0, \ldots, 0) & \text { otherwise. }\end{cases}
$$

Observe that $\mu \in R_{k}\left(\mathcal{A}_{Y}\right) \subset \bigoplus_{X \in L_{k-1}^{Y}} R_{k-1}\left(\mathcal{A}_{X}\right)$. Recall that, for $X \in$ $L_{k-1}^{Y}$, the notation $\mu_{X}$ denotes the $X$ coordinate of $\mu$. Now we may define

$$
\omega_{\mu}(B)=\frac{1}{\beta_{1}} \sum_{X \in L_{k-1}^{Y}} \omega_{\mu_{X}}\left(C^{X}\right) .
$$

If $k=2$, there is a convenient alternative formula for $\omega_{\mu}$. In this case, $B=$ $\left(\beta_{1}, \beta_{2}\right)$ for some $\beta_{1}, \beta_{2} \in Y^{\perp}$. Observe $\mu \in R_{2}\left(\mathcal{A}_{Y}\right) \subset \bigoplus_{X \in L_{1}^{Y}} R_{1}\left(\mathcal{A}_{X}\right)$. We may write $\mu=\sum_{X \in \mathcal{A}_{Y}} c_{X} e_{X}$, where $c_{X} \in \mathbf{K}$. For every $X \in \mathcal{A}_{Y}$, write $\alpha_{X}=\alpha_{X 1} \beta_{1}+\alpha_{X 2} \beta_{2}$ with $\alpha_{X 1}, \alpha_{X 2} \in \mathbf{K}$. Then

$$
\omega_{\mu}(B)=\frac{1}{\beta_{1}} \sum_{X \in \mathcal{A}_{Y}} c_{X} \alpha_{X 2} \frac{d \alpha_{X}}{\alpha_{X}} .
$$

\section{Properties.}

We prove several properties of the forms constructed in the previous section, including the property that these forms are logarithmic. In Theorem 6.7 it is shown that these forms are, up to scalar multiple, independent of the tuple $B$.

First we prove a technical lemma. Let $2<k \leq l, Y \in L_{k}$, and $\mu \in$ $R_{k}\left(A_{Y}\right)$. Let $B=\left(\beta_{1}, \ldots, \beta_{k}\right)$ be a basis of $Y^{\perp}$. To simplify notation, for any $X \in L_{k-1}, Z \in L_{k-2}$, write $\mu_{X}^{Z}=\left(\mu_{X}\right)_{Z}$.

For any $Z \in L_{k-2}^{Y}$, if $Z^{\perp} \cap\left\langle\beta_{1}, \beta_{2}\right\rangle=0$, then let $C^{Z}$ be the basis of $Z$ given by Lemma 5.1 so that $\left.C^{Z}\right|_{V\left(\beta_{1}, \beta_{2}\right)}=\left.\left(\beta_{3}, \ldots, \beta_{k}\right)\right|_{V\left(\beta_{1}, \beta_{2}\right)}$. 
Extend this definition to all $Z \in L_{k-2}^{Y}$ via

$$
C^{Z}= \begin{cases}C^{Z} & \text { (as above) if } Z^{\perp} \cap\left\langle\beta_{1}, \beta_{2}\right\rangle=0 \\ (0, \ldots, 0) & \text { otherwise. }\end{cases}
$$

Lemma 6.1. We have the identity

$$
\beta_{1} \beta_{2} \omega_{\mu}(B)=\sum_{\substack{X \in L_{k-1}^{Y} \\ \beta_{1} \notin X^{\perp}}} \sum_{Z \in L_{k-2}^{Y}} \frac{\beta_{2}}{\gamma_{2}^{X}} \omega_{\mu_{X}^{Z}}\left(C^{Z}\right) .
$$

Proof. First, for any $X \in L_{k-1}^{Y}, \beta_{1} \notin X^{\perp}$, and $Z \in L_{k-2}^{X}$ with $\gamma_{2}^{X} \notin Z^{\perp}$, Lemma 5.1 gives a unique basis $C^{X, Z}$ with $\left.C^{X, Z}\right|_{V\left(\gamma_{2}^{X}\right)}=\left.\left(\gamma_{3}^{X}, \ldots, \gamma_{k}^{X}\right)\right|_{V\left(\gamma_{2}^{X}\right)}$. Extend this definition to all $X \in L_{k-1}^{Y}$ with $\beta_{1} \notin X^{\perp}$ and $Z \in L_{k-2}^{Y}$ via

$$
C^{X, Z}= \begin{cases}C^{X, Z} & \text { (as above) if } Z \in L_{k-2}^{X} \text { and } \gamma_{2}^{X} \notin Z^{\perp} \\ (0, \ldots, 0) & \text { otherwise. }\end{cases}
$$

Observe that, for any $X \in L_{k-2}^{X}$ with $\beta_{1} \notin X^{\perp}$, the definition of $\omega_{\mu_{X}}\left(C^{X}\right)$ gives us

$$
\omega_{\mu_{X}}\left(C^{X}\right)=\frac{1}{\gamma_{1}^{X}} \sum_{Z \in L_{k-2}^{Y}} \omega_{\mu_{X}^{Z}}\left(C^{X, Z}\right) .
$$

By definition of $C^{Z}$ and $C^{X, Z}$, we have $C^{X, Z}=C^{Z}$ for every $X \in L_{k-1}^{Y}$ with $\beta_{1} \notin X^{\perp}, Z \in L_{k-2}^{X}$.

Now we are ready to prove the equation. By definition,

$$
\begin{aligned}
\beta_{1} \beta_{2} \omega_{\mu}(B) & =\beta_{2} \sum_{\substack{X \in L_{k-1}^{Y}\\
}} \omega_{\mu_{X}}\left(C^{X}\right) \\
& =\beta_{2} \sum_{\substack{X \in L_{k-1}^{Y} \\
\beta_{1} \notin X^{\perp}}} \frac{1}{\gamma_{2}^{X}} \sum_{Z \in L_{k-2}^{X}} \omega_{\mu_{X}^{Z}}\left(C^{X, Z}\right) \\
& =\sum_{\substack{X \in L_{k-1}^{Y} \\
\beta_{1} \notin X^{\perp}}} \sum_{Z \in L_{k-2}^{X}} \frac{\beta_{2}}{\gamma_{2}^{X}} \omega_{\mu_{X}^{Z}}\left(C^{Z}\right) .
\end{aligned}
$$

If $Z \in L_{k-2}^{Y} \backslash L_{k-2}^{X}$, then $\mu_{X}^{Z}=0$ and $\omega_{\mu_{X}^{Z}}\left(C^{Z}\right)=0$, so the last expression is

$$
\sum_{\substack{X \in L_{k-1}^{Y} \\ \beta_{1} \notin X^{\perp}}} \sum_{\substack{Z \\ Z \in L_{k-2}^{Y}}} \frac{\beta_{2}}{\gamma_{2}^{X}} \omega_{\mu_{X}^{Z}}\left(C^{Z}\right)
$$


The following lemma is well-known. It is needed in Lemma 6.3 to establish a necessary and sufficient condition for certain rational differential 1-forms to be logarithmic.

Lemma 6.2. Let $\omega$ be a global rational differential $p$-form on $V$ such that $Q \omega \in \Omega^{p}[V]$. Then $\omega \in \Omega^{p}(\mathcal{A})$ if and only if $Q\left(d \alpha_{X} / \alpha_{X}\right) \wedge \omega \in \Omega^{p+1}[V]$ for every $X \in \mathcal{A}$.

Proof. See [OT, Proposition 4.78].

Lemma 6.3. Let $\omega \in \Omega^{1}(\mathcal{A})$ and $X \in \mathcal{A}$. Let $\rho$ denote restriction to $X$. Assume that $\alpha_{X}$ divides $Q \omega$ in the $S$-module $\Omega^{1}[V]$. Then $\frac{1}{\alpha_{X}} \omega \in \Omega^{1}(\mathcal{A})$ if and only if $\rho\left(\frac{Q}{\alpha_{X}} \omega\right)=0$.

Proof. Since $\alpha_{X}$ divides $Q \omega$ in $\Omega^{1}[V]$, we have $Q \frac{1}{\alpha_{X}} \omega \in \Omega^{1}[V]$ and we may apply Lemma 6.2.

Choose a basis $\left(x_{1}, x_{2}, \ldots, x_{l}\right)$ of $V^{*}$ with $x_{1}=\alpha_{1}$. For every $i, 1 \leq i \leq l$, let $\frac{p_{i}}{q_{i}}$ represent the $d x_{i}$ coefficient of $\omega$, where $p_{i}$ and $q_{i}$ are chosen to be relatively prime elements of $S=\mathbf{K}\left[x_{1}, \ldots, x_{l}\right]$, with $q_{i}$ monic.

Assume $\frac{1}{x_{1}} \omega \in \Omega^{1}(\mathcal{A})$. Then by Lemma 6.2,

$$
Q \frac{d x_{1}}{x_{1}} \wedge \frac{1}{x_{1}} \omega=\sum_{i=2}^{l} Q \frac{p_{i}}{q_{i} x_{1}^{2}} d x_{1} \wedge d x_{i} \in \Omega^{2}[V] .
$$

Let $i, 2 \leq i \leq l$ be arbitrary. Since $\Omega^{2}[V]$ is a free $S$-module with basis $\left\{d x_{j} \wedge d x_{k} \mid 1 \leq j<k \leq l\right\}$ we have $Q \frac{p_{i}}{q_{i} x_{1}^{2}} d x_{1} \wedge d x_{i} \in \Omega^{2}[V]$. Therefore $Q \frac{p_{i}}{q_{i} x_{1}^{2}}$ is polynomial. Thus $x_{1}$ divides $Q \frac{p_{i}}{q_{i} x_{1}}$ in the ring $S$, and so $\rho\left(Q \frac{p_{i}}{q_{i} x_{1}}\right)=0$. Thus

$$
\rho\left(\frac{Q}{x_{1}} \omega\right)=\rho\left(Q \frac{p_{1}}{q_{1} x_{1}} d x_{1}\right)+\rho\left(\sum_{i=2}^{l} Q \frac{p_{i}}{q_{i} x_{1}} d x_{i}\right)=0 .
$$

Conversely, assume $\rho\left(\frac{Q}{x_{1}} \omega\right)=0$. We have

$$
\rho\left(\frac{Q}{x_{1}} \omega\right)=\sum_{i=2}^{l} \rho\left(Q \frac{p_{i}}{q_{i} x_{1}}\right) d \rho\left(x_{i}\right) .
$$

Since $\Omega^{1}\left[V\left(x_{1}\right)\right]$ is a free $K\left[\rho\left(x_{2}\right), \ldots, \rho\left(x_{l}\right)\right]$ module with basis $\left\{d \rho\left(x_{2}\right), \ldots\right.$, $\left.d \rho\left(x_{l}\right)\right\}, \rho\left(Q \frac{p_{i}}{q_{i} x_{1}}\right)=0$ for $i=2, \ldots, l$. Therefore, $x_{1}$ divides $Q \frac{p_{i}}{q_{i} x_{1}}$ in the ring $S$. Thus $Q \frac{p_{i}}{q_{i} x_{1}^{2}} \in S$ and so $Q \frac{p_{i}}{q_{i} x_{1}^{2}} d x_{1} \wedge d x_{i} \in \Omega[V]$. Therefore $Q \frac{d x_{1}}{x_{1}} \wedge$ $\frac{1}{x_{1}} \omega=\sum_{i=2}^{l} Q \frac{p_{i}}{q_{i} x_{1}^{2}} d x_{1} \wedge d x_{i}$ is regular. By Lemma 6.2, $\frac{1}{x_{1}} \omega$ is logarithmic. 
Lemma 6.4. For $\nu, v \in R_{k}\left(\mathcal{A}_{Y}\right)$ and $a, b \in \mathbf{K}$,

$$
a \omega_{\nu}(B)+b \omega_{v}(B)=\omega_{a \nu+b v}(B) .
$$

That is, the assignment $\mu \rightarrow \omega_{\mu}$ is $\mathbf{K}$-linear.

Proof. This is obvious for $k=1$, and follows immediately for arbitrary $k$ by induction.

The following theorem is our main result. It is a list of properties of $\omega_{\mu}(B)$, with property (iii) being the most important.

Notation. For Theorem 6.5 and its proof, we make the following notational conventions. The symbol $\theta$ will represent an element of $V^{*}, \rho$ will denote restriction of differential forms to $V(\theta)$, and $\mathcal{A}^{\prime \prime}$ will denote the restriction of $\mathcal{A}$ to $V(\theta)$. For $1 \leq i \leq l$, let $h_{i}$ be the linear map defined in Sections 3 and 4. For $X \in L, \alpha \in V^{*}$, and $Z \in \mathcal{A}^{\prime \prime}$, let $X^{\prime \prime}, \alpha^{\prime \prime}$, and $\eta_{Z}$ be as in Section 3 .

Theorem 6.5. Let $k \geq 2, Y \in L_{k}$, and $\mu \in R_{k}\left(\mathcal{A}_{Y}\right)$. Write $\omega_{\mu}=\omega_{\mu}(B)$.

(i) For any permutation $\sigma$ of $(1, \ldots, k)$,

$$
\omega_{\mu}\left(\beta_{\sigma(1)}, \ldots, \beta_{\sigma(k)}\right)=(\operatorname{sgn} \sigma) \omega_{\mu} .
$$

(ii) Let $\eta=b_{1} \beta_{1}+\ldots+b_{k} \beta_{k}$, where each $b_{i} \in \mathbf{K}$ and $b_{1} \neq 0$. Then

$$
b_{1} \omega_{\mu}\left(\eta, \beta_{2}, \ldots, \beta_{k}\right)=\omega_{\mu} \text {. }
$$

(iii) $\omega_{\mu} \in \Omega\left(\mathcal{A}_{Y}\right)$.

(iv) Assume $\theta \notin Y^{\perp}$. Then

$$
\rho\left(\omega_{\mu}\right)=\omega_{h_{k-1}^{Y} \mu}\left(\beta_{1}^{\prime \prime}, \ldots, \beta_{k}^{\prime \prime}\right) .
$$

Proof. First we prove (i)-(iv) in the case $k=2$. Write $\mu=\sum_{X \in \mathcal{A}_{Y}} c_{X} e_{X}$, and use Equation (6) to write $\omega_{\mu}=\frac{1}{\beta_{1}} \sum_{X \in \mathcal{A}_{Y}} c_{X} \alpha_{X 2} \frac{d \alpha_{X}}{\alpha_{X}}$.

(i) Since $k=2, \sigma$ is either the identity or the transposition $(1,2)$. If $\sigma=$ identity, the claim is obvious. If $\sigma=(1,2)$, the claim is $\omega_{\mu}=-\omega_{\mu}\left(\beta_{2}, \beta_{1}\right)$. By definition of $R_{2}\left(\mathcal{A}_{Y}\right), \delta_{1}^{Y} \mu=0$. However, $\delta_{1}^{Y}(\mu)=\sum_{X \in \mathcal{A}_{Y}} c_{X} \alpha_{X}$, so

$$
\sum_{X \in \mathcal{A}_{Y}} c_{X} \alpha_{X}=0 .
$$

Therefore

$$
\begin{aligned}
\beta_{1} \beta_{2}\left(\omega_{\mu}\left(\beta_{2}, \beta_{1}\right)+\omega_{\mu}\right) & =\sum_{X \in \mathcal{A}_{Y}} c_{X} \alpha_{X 1} \beta_{1} \frac{d \alpha_{X}}{\alpha_{X}}+\sum_{X \in \mathcal{A}_{Y}} c_{X} \alpha_{X 2} \beta_{2} \frac{d \alpha_{X}}{\alpha_{X}} \\
& =\sum_{X \in \mathcal{A}_{Y}} c_{X} d \alpha_{X} \\
& =0 .
\end{aligned}
$$


There are no zero divisors in the $S$-module of global rational 1-forms on $V$. Since $\beta_{1} \beta_{2} \neq 0$, we have $\omega_{\mu}\left(\beta_{2}, \beta_{1}\right)+\omega_{\mu}=0$, proving the claim.

(ii) Since $b_{1} \neq 0,\left(\eta, \beta_{2}\right)$ is a basis of $Y^{\perp}$. For any $\alpha_{X} \in \mathcal{A}_{Y}$, write

$$
\alpha_{X}=\alpha_{X 1}^{\prime} \eta+\alpha_{X 2}^{\prime} \beta_{2} .
$$

Then $\alpha_{X 1}=b_{1} \alpha_{X 1}^{\prime}$. Therefore

$$
\begin{aligned}
b_{1} \omega_{\mu}\left(\eta, \beta_{2}\right) & =-b_{1} \omega_{\mu}\left(\beta_{2}, \eta\right) \\
& =-\frac{1}{\beta_{2}} \sum_{X \in \mathcal{A}_{Y}} c_{X} \alpha_{X 1} \frac{d \alpha_{X}}{\alpha_{X}} \\
& =-\omega_{\mu}\left(\beta_{2}, \beta_{1}\right) \\
& =\omega_{\mu}\left(\beta_{1}, \beta_{2}\right)
\end{aligned}
$$

which proves property (ii).

(iii) Without loss of generality we may assume that $V\left(\beta_{1}\right) \in \mathcal{A}_{Y}$. Indeed, choose any $X \in \mathcal{A}_{Y}$ so that $X \neq V\left(\beta_{2}\right)$. Then, writing $\alpha_{X}=\alpha_{X 1} \beta_{1}+\alpha_{X 2} \beta_{2}$, we have $\alpha_{X 1} \neq 0$. By (ii), $\alpha_{X 1} \omega\left(\alpha_{X}, \beta_{2}\right)=\omega\left(\beta_{1}, \beta_{2}\right)$. Since $\Omega^{1}(\mathcal{A})$ is closed under scalar multiplication, it suffices to show $\omega\left(\alpha_{X}, \beta_{2}\right) \in \Omega^{1}(\mathcal{A})$. Thus we may and shall assume $V\left(\beta_{1}\right) \in \mathcal{A}_{Y}$.

Since for every $X \in \mathcal{A}_{Y}, \frac{d \alpha_{X}}{\alpha_{X}} \in \Omega\left(\mathcal{A}_{Y}\right)$, the sum $\sum_{X \in \mathcal{A}_{Y}} c_{X} \alpha_{X 2} \frac{d \alpha_{X}}{\alpha_{X}}$ is in the module $\Omega\left(\mathcal{A}_{Y}\right)$. We now check that $\beta_{1}$ divides $Q_{Y} \sum_{X \in \mathcal{A}_{Y}} c_{X} \alpha_{X 2} \frac{d \alpha_{X}}{\alpha_{X}}$ in the $S$-module $\Omega^{1}[V]$ so that we may apply Lemma 6.3. Since $V\left(\beta_{1}\right) \in \mathcal{A}_{Y}$, $\beta_{1}$ divides $Q_{Y}$. For any $\alpha_{X} \in \mathcal{A}_{Y}$ with $\alpha_{X} \neq \beta_{1}, \alpha_{X}$ is nonproportional to $\beta_{1}$, so $\alpha_{X}$ and $\beta_{1}$ are not associates in the unique factorization domain $S$. Thus $\beta_{1}$ divides $\frac{Q_{Y}}{\alpha_{X}}$ in $S$, so $\beta_{1}$ divides $Q_{Y} c_{X} \alpha_{X 2} \frac{d \alpha_{X}}{\alpha_{X}}$ in $\Omega^{1}[V]$. Therefore, $\beta_{1}$ divides each summand of

$$
\begin{aligned}
Q_{Y} \sum_{X \in \mathcal{A}_{Y}} c_{X} \alpha_{X 2} \frac{d \alpha_{X}}{\alpha_{X}} & =\sum_{X \in \mathcal{A}_{Y}} Q_{Y} c_{X} \alpha_{X 2} \frac{d \alpha_{X}}{\alpha_{X}} \\
& =\sum_{\substack{X \in \mathcal{A}_{Y} \\
\alpha_{X} \neq \beta_{1}}} Q_{Y} c_{X} \alpha_{X 2} \frac{d \alpha_{X}}{\alpha_{X}}
\end{aligned}
$$

in $\Omega^{1}[V]$, and so $\beta_{1}$ divides $Q_{Y} \sum_{X \in \mathcal{A}_{Y}} c_{X} \alpha_{X 2} \frac{d \alpha_{X}}{\alpha_{X}}$.

Let $\rho_{1}$ denote restriction to $V\left(\beta_{1}\right)$. By Lemma 6.3, it suffices to show

$$
\begin{aligned}
\rho_{1}\left(Q_{Y} \omega_{\mu}\right) & =\rho_{1}\left(\frac{Q_{Y}}{\beta_{1}} \sum_{X \in \mathcal{A}_{Y}} c_{X} \alpha_{X 2} \frac{d \alpha_{X}}{\alpha_{X}}\right) \\
& =0 .
\end{aligned}
$$


By definition, $\mu \in \operatorname{ker} \delta_{1}^{Y}$. But

$$
\begin{aligned}
\delta_{1}^{Y \mu} & =\sum_{X \in \mathcal{A}_{Y}} c_{X} \alpha_{X} \\
& =\sum_{X \in \mathcal{A}_{Y}} c_{X}\left(\alpha_{X 1} \beta_{1}+\alpha_{X 2} \beta_{2}\right) \\
& =\left(\sum_{X \in \mathcal{A}_{Y}} c_{X} \alpha_{X 1}\right) \beta_{1}+\left(\sum_{X \in \mathcal{A}_{Y}} c_{X} \alpha_{X 2}\right) \beta_{2} .
\end{aligned}
$$

Since $\left(\beta_{1}, \beta_{2}\right)$ is a basis of $Y^{\perp}, \sum_{X \in \mathcal{A}_{Y}} c_{X} \alpha_{X 2}=0$.

Thus

$$
\begin{aligned}
\rho_{1}\left(\frac{Q_{Y}}{\beta_{1}} \sum_{X \in \mathcal{A}_{Y}} c_{X} \alpha_{X 2} \frac{d \alpha_{X}}{\alpha_{X}}\right) & =\rho_{1}\left(\frac{Q_{Y}}{\beta_{1}}\right)\left(\sum_{\substack{X \in \mathcal{A}_{Y} \\
X \neq V\left(\beta_{1}\right)}} c_{X} \alpha_{X 2}\right) \frac{d \rho_{1}\left(\beta_{2}\right)}{\rho_{1}\left(\beta_{2}\right)} \\
& =0 .
\end{aligned}
$$

Therefore, by Lemma $6.3, \omega_{\mu}=\frac{1}{\beta_{1}} \sum_{X \in \mathcal{A}} c_{X} \alpha_{X 2} \frac{d \alpha_{X}}{\alpha_{X}} \in \Omega\left(\mathcal{A}_{Y}\right)$.

(iv) For any $Z \in\left(\mathcal{A}_{Y}\right)^{\prime \prime}$, write

$$
\eta_{Z}=\eta_{Z 1} \beta_{1}^{\prime \prime}+\eta_{Z 2} \beta_{2}^{\prime \prime}
$$

For $X \in \mathcal{A}_{Y}$, write $\alpha_{X}^{\prime \prime}=\alpha_{X 1} \beta_{1}^{\prime \prime}+\alpha_{X 2} \beta_{2}^{\prime \prime}$, and note that

$$
\alpha_{X 2} \eta_{X^{\prime \prime}}=\eta_{X^{\prime \prime} 2} \alpha_{X}^{\prime \prime}
$$

i.e.

$$
\frac{\alpha_{X}^{\prime \prime}}{\eta_{X^{\prime \prime}}} \eta_{X^{\prime \prime 2}}=\alpha_{X 2}
$$

Now recall that $h_{1}^{Y} \mu=\sum_{X \in \mathcal{A}_{Y}} c_{X} \frac{\alpha_{X}^{\prime \prime}}{\eta_{X^{\prime \prime}}} e_{X^{\prime \prime}}$, so

$$
\begin{aligned}
\omega_{h_{1}^{Y} \mu}\left(\beta_{1}^{\prime \prime}, \ldots, \beta_{k}^{\prime \prime}\right) & =\frac{1}{\beta_{1}^{\prime \prime}} \sum_{X \in \mathcal{A}_{Y}} c_{X} \frac{\alpha_{X}^{\prime \prime}}{\eta_{X^{\prime \prime}}} \eta_{X^{\prime \prime}} \frac{d \eta_{X^{\prime \prime}}}{\eta_{X^{\prime \prime}}} \\
& =\frac{1}{\beta_{1}^{\prime \prime}} \sum_{X \in \mathcal{A}_{Y}} c_{X} \alpha_{X 2} \frac{d \alpha_{X}^{\prime \prime}}{\alpha_{X}^{\prime \prime}} \\
& =\rho\left(\omega_{\mu}\right) .
\end{aligned}
$$

This proves property (iv).

Now we prove properties (i)-(iv) in the case when $k>2$.

(i) Step 1. We claim $\omega_{\mu}\left(\beta_{2}, \beta_{1}, \beta_{3}, \ldots, \beta_{k}\right)=-\omega_{\mu}$, i.e., the property holds if $\sigma$ is the transposition $(1,2)$.

For $X \in L_{k-1}^{Y}, \beta_{1} \notin X^{\perp}$, let $a_{i}^{X}$ be as in Lemma 5.2. Then $C^{X}=$ $\left(\gamma_{2}^{X}, \ldots, \gamma_{k}^{X}\right)$ has $\gamma_{i}=\beta_{i}-a_{i}^{X} \beta_{1}$ for each $i$. Now consider the basis $\left(\beta_{2}, \beta_{1}, \beta_{3}, \beta_{4}, \ldots, \beta_{k}\right)$ of $Y^{\perp}$. For any $X \in L_{k-1}^{Y}$ with $\beta_{2} \notin X^{\perp}$, let $D^{X}$ be 
the unique basis of $X^{\perp}$ given by Lemma 5.1 with $\left.D^{X}\right|_{V\left(\beta_{2}\right)}=\left(\beta_{1}, \beta_{3}, \beta_{4}, \ldots\right.$, $\left.\beta_{k}\right)\left.\right|_{V\left(\beta_{2}\right)}$. Then for each $i, i=1,3,4, \ldots, k$, let $b_{i}^{X}$ be the scalar with $b_{i}^{X} \beta_{2} \equiv \beta_{i} \quad\left(\bmod X^{\perp}\right)$. Lemma 5.2 applied in this situation shows

$$
D^{X}=\left(\beta_{1}-b_{1}^{X} \beta_{2}, \beta_{3}-b_{3}^{X} \beta_{2}, \beta_{4}-b_{4}^{X} \beta_{2}, \ldots, \beta_{k}-b_{k}^{X} \beta_{2}\right) .
$$

Now apply Lemma 6.1, obtaining

$$
\beta_{1} \beta_{2} \omega_{\mu}(B)=\sum_{\substack{X \in L_{k-1}^{Y} \\ \beta_{1} \notin X^{\perp}}} \sum_{Z \in L_{k-2}^{Y}} \frac{\beta_{2}}{\beta_{2}-a_{2}^{X} \beta_{1}} \omega_{\mu_{X}^{Z}}\left(C^{Z}\right) .
$$

Another application of the same lemma yields

$$
\beta_{1} \beta_{2} \omega_{\mu}\left(\beta_{2}, \beta_{1}, \beta_{3}, \ldots, \beta_{k}\right)=\sum_{\substack{X \in L_{k-1}^{Y} \\ \beta_{2} \notin X^{\perp}}} \sum_{Z \in L_{k-2}} \frac{\beta_{1}}{\beta_{1}-b_{1}^{X} \beta_{2}} \omega_{\mu_{X}^{Z}}\left(C^{Z}\right) .
$$

Now notice that for any $X \in L_{k-1}^{Y}$ with $\beta_{1} \notin X^{\perp}, \beta_{2} \notin X^{\perp}$, we have $b_{1}^{X}=\frac{1}{a_{2}^{X}}$ by definition of these scalars. Also note that, for $X$ with $\beta_{1} \notin X^{\perp}$, $a_{2}^{X}=0$ if and only if $\beta_{2} \in X^{\perp}$ and for $X$ with $\beta_{2} \notin X^{\perp}, b_{1}^{X}=0$ if and only if $\beta_{1} \in X^{\perp}$. Together, these observations imply that

$$
\beta_{1} \beta_{2}\left(\omega_{\mu}\left(\beta_{2}, \beta_{1}, \beta_{3}, \ldots, \beta_{k}\right)+\omega_{\mu}\right)=\sum_{Z \in L_{k-2}^{Y}} \sum_{X \in L_{k-1}^{Y}} \omega_{\mu_{X}^{Z}}\left(C^{Z}\right) .
$$

By Lemma 6.4, the last expression is

$$
\sum_{Z \in L_{k-2}^{Y}} \omega_{\diamond}\left(C^{Z}\right)
$$

where $\diamond=\sum_{X \in L_{k-1}^{Y}} \mu_{X}^{Z}$. Now, by definition of $\delta_{k-1}^{Y}, \diamond=\left(\delta_{k-1}^{Y} \mu\right)_{Z}$. However, $\delta_{k-1}^{Y} \mu=0$. So by definition, each $\omega_{\diamond}\left(C^{Z}\right)=0$. Therefore

$$
\beta_{1} \beta_{2}\left(\omega_{\mu}\left(\beta_{2}, \beta_{1}, \beta_{3}, \ldots, \beta_{k}\right)+\omega_{\mu}\right)=0 .
$$

Since $\beta_{1} \beta_{2}$ is nonzero,

$$
\omega_{\mu}\left(\beta_{2}, \beta_{1}, \beta_{3}, \ldots, \beta_{k}\right)+\omega_{\mu}=0
$$

i.e., $\omega_{\mu}\left(\beta_{2}, \beta_{1}, \beta_{3}, \ldots, \beta_{k}\right)=-\omega_{\mu}$. This proves the claim in Step 1.

Step 2. We now show, for any $q>1$,

$$
\omega_{\mu}\left(\beta_{1}, \beta_{2}, \ldots, \beta_{q-1}, \beta_{q+1}, \beta_{q}, \beta_{q+2} \ldots, \beta_{k}\right)=-\omega_{\mu},
$$

that is, the claim in (i) holds when $\sigma$ is the transposition $(q, q+1)$. 
Proof of Step 2: Let $C^{X}=\left(\gamma_{2}^{X}, \ldots, \gamma_{k}^{X}\right)$ be as in Lemma 5.1. Then by the inductive hypothesis,

$$
\begin{aligned}
\omega_{\mu} & =\frac{1}{\beta_{1}} \sum \omega_{\mu_{X}}\left(C^{X}\right) \\
& =-\frac{1}{\beta_{1}} \sum \omega_{\mu_{X}}\left(\gamma_{2}^{X}, \ldots, \gamma_{q+1}^{X}, \gamma_{q}^{X}, \ldots, \gamma_{k}^{X}\right) \\
& =-\omega_{\mu}\left(\beta_{1}, \beta_{2}, \ldots, \beta_{q+1}, \beta_{q}, \ldots, \beta_{k}\right) .
\end{aligned}
$$

Step 3. We now show $\omega_{\mu}\left(\beta_{\sigma(1)}, \ldots, \beta_{\sigma(k)}\right)=\operatorname{sgn} \sigma \omega_{\mu}$.

Write $\sigma$ as a product $\sigma_{N} \circ \sigma_{N-1} \circ \cdots \circ \sigma_{1}$ of transpositions $\sigma_{i}$ with the following property.

Let $\tau_{i}=\sigma_{i-1} \circ \cdots \circ \sigma_{1}$ for $i>1$ and $\tau_{1}$ be the identity. Then for every $i, 1 \leq i \leq N, \sigma_{i}$ switches the positions of two adjacent entries in the tuple $\left(\tau_{i}(1), \ldots, \tau_{i}(k)\right)$.

By Steps 1 and 2,

$$
\begin{aligned}
\omega_{\mu}\left(\beta_{\sigma(1)}, \ldots, \beta_{\sigma(k)}\right) & =\omega_{\mu}\left(\beta_{\tau_{N+1}(1)}, \ldots, \beta_{\tau_{N+1}(k)}\right) \\
& =-\omega_{\mu}\left(\beta_{\tau_{N}(1)}, \ldots, \beta_{\tau_{N}(k)}\right) \\
& =\cdots \\
& =(-1)^{N} \omega_{\mu} \\
& =\operatorname{sgn} \sigma \omega_{\mu} .
\end{aligned}
$$

(ii) By property (i), Lemma 5.2, and induction we have, for some $s_{\eta}^{X}, s_{1}^{X}$, $s_{3}^{X}, \cdots s_{k}^{X} \in \mathbf{K}$,

$$
\begin{aligned}
b_{1} \omega_{\mu}\left(\eta, \beta_{2}, \ldots, \beta_{k}\right) & =-b_{1} \omega_{\mu}\left(\beta_{2}, \eta, \beta_{3} \ldots, \beta_{k}\right) \\
& =-\frac{b_{1}}{\beta_{2}} \sum_{X \in L_{k-1}} \omega_{\mu}\left(\eta-s_{\eta}^{X} \beta_{2}, \beta_{3}-s_{3}^{X} \beta_{2}, \ldots, \beta_{k}-s_{k}^{X} \beta_{2}\right) \\
& =-\frac{1}{\beta_{2}} \sum_{X \in L_{k-1}} \omega_{\mu}\left(\beta_{1}-s_{1}^{X} \beta_{2}, \beta_{3}-s_{3}^{X} \beta_{2} \ldots, \beta_{k}-s_{k}^{X} \beta_{2}\right) \\
& =\omega_{\mu} .
\end{aligned}
$$

(iii) We may assume without loss of generality that $V\left(\beta_{1}\right) \in \mathcal{A}_{Y}$. Indeed, take any $X \in \mathcal{A}_{Y}$ with $\alpha_{X 1} \neq 0$, where $\alpha_{X}=\alpha_{X 1} \beta_{1}+\alpha_{X 2} \beta_{2}+$ $\cdots+\alpha_{X k} \beta_{k}$. Then by (ii), $\omega_{\mu}\left(\alpha_{X}, \beta_{2}, \ldots, \beta_{k}\right) \doteq \omega_{\mu}$. Thus it suffices to show $\omega_{\mu}\left(\alpha_{X}, \beta_{2}, \ldots, \beta_{k}\right) \in \Omega(\mathcal{A})$. Thus we may and shall assume that $V\left(\beta_{1}\right) \in \mathcal{A}_{Y}$.

By the inductive hypothesis (iii), $Q_{X} \omega_{\mu_{X}}\left(C^{X}\right) \in \Omega^{1}[V]$ for each $X \in$ $L_{k-1}^{Y}$. For any $X \in L_{k-1}^{Y}$ with $\beta_{1} \notin X^{\perp}$, we have $\beta_{1} \notin \mathcal{A}_{X}$ so $\beta_{1}$ does not divide $Q_{X}$ in $S$. However, $\beta_{1}$ divides $Q_{Y}$ so $\beta_{1}$ divides $\frac{Q_{Y}}{Q_{X}}$. Thus $\beta_{1}$ divides 
$\frac{Q_{Y}}{Q_{X}} Q_{X} \omega_{\mu_{X}}\left(C^{X}\right)=Q_{Y} \omega_{\mu_{X}}\left(C^{X}\right)$ in $\Omega^{1}[V]$. Any $X$ with $\beta_{1} \in X^{\perp}$ has $\mu_{X}=0$ so $\beta_{1}$ divides

$$
Q_{Y} \sum_{X \in L_{k-1}^{Y}} \omega_{\mu_{X}}\left(C^{X}\right)=Q_{Y} \sum_{\substack{X \in L_{k-1}^{Y} \\ \beta_{1} \notin X^{\perp}}} \omega_{\mu_{X}}\left(C^{X}\right)=\sum_{\substack{X \in L_{k-1}^{Y} \\ \beta_{1} \notin X^{\perp}}} Q_{Y} \omega_{\mu_{X}}\left(C^{X}\right)
$$

in $\Omega^{1}[V]$. Thus by Lemma 6.2 it suffices to check that the restriction of $Q_{Y} \omega_{\mu}=\frac{Q_{Y}}{\beta_{1}} \sum_{X \in L_{k-1}^{Y}} \omega_{\mu_{X}}\left(C^{X}\right)$ to $V\left(\beta_{1}\right)$ is 0 .

To see this, we apply the inductive hypothesis in the case $\theta=\beta_{1}$. Note that for $X \in L_{k-1}^{Y}$ with $\beta_{1} \notin X^{\perp}$, and $i \geq 2,\left(\gamma_{i}^{X}\right)^{\prime \prime}=\beta_{i}^{\prime \prime}$ by definition. Also note that for $X \in L_{k-1}^{Y}$ with $\beta_{1} \notin X^{\perp}, X^{\prime \prime}=Y$.

By Lemma 6.4 we have

$$
\begin{aligned}
\rho_{1}\left(\frac{Q_{Y}}{\beta_{1}} \sum_{X \in L_{k-1}^{Y}} \omega_{\mu_{X}}\left(C_{X}\right)\right) & =\rho_{1}\left(\frac{Q_{Y}}{\beta_{1}} \sum_{\substack{X \in L_{k-1}^{Y} \\
\beta \notin X^{\perp}}} \omega_{\mu_{X}}\left(C_{X}\right)\right) \\
& =\rho_{1}\left(\frac{Q_{Y}}{\beta_{1}}\right) \sum_{\substack{X \in L_{k-1}^{Y} \\
\beta \notin X^{\perp}}} \omega_{h_{k-2}^{X} \mu_{X}}\left(\beta_{2}^{\prime \prime}, \ldots, \beta_{k}^{\prime \prime}\right) \\
& =\rho_{1}\left(\frac{Q_{Y}}{\beta_{1}}\right) \omega_{\diamond}\left(\beta_{2}^{\prime \prime}, \ldots, \beta_{2}^{\prime \prime}\right) .
\end{aligned}
$$

Where $\diamond=\sum_{X \in L_{k-1}^{Y}} h_{k-2}^{X} \mu_{X}$. This is 0 by Lemma 4.3.

Thus we have shown

$$
\rho_{1}\left(Q_{Y} \omega_{\mu}\right)=\omega_{0}\left(\beta_{2}^{\prime \prime}, \ldots, \beta_{2}^{\prime \prime}\right)=0 .
$$

This proves (iii).

(iv) To simplify notation, we write $\gamma_{i}^{X \prime \prime}$ to stand for $\left(\gamma_{i}^{X}\right)^{\prime \prime}$. For any $Z \in$ $L^{\prime \prime}$, let $Z_{V(\theta)}^{\perp}$ represent the orthogonal complement of $Z$ in $V(\theta)^{*}$. Observe that since $\theta \notin Y^{\perp}$, the codimension of $Y^{\prime \prime}$ in $V(\theta)$ is $k$, and $\beta_{1}^{\prime \prime}, \ldots, \beta_{k}^{\prime \prime}$ form a basis for $\left(Y^{\prime \prime}\right)_{V(\theta)}^{\perp}$.

Recall that $\left(\mathcal{A}_{Y}\right)^{\prime \prime} \subset \mathcal{A}_{Y^{\prime \prime}}^{\prime \prime}$, and in particular, $L\left(\left(\mathcal{A}_{Y}\right)^{\prime \prime}\right) \subset L\left(\mathcal{A}_{Y^{\prime \prime}}^{\prime \prime}\right)$. Thus for $Z \in L_{k-1}\left(\left(\mathcal{A}_{Y}\right)^{\prime \prime}\right)$ with $\beta_{1}^{\prime \prime} \notin Z_{V(\theta)}^{\perp}$, Lemma 5.1 gives us a basis $E^{Z}$ of elements in $Z_{V(\theta)}^{\perp}$ with $\left.E^{Z}\right|_{\text {ker } \beta_{1}^{\prime \prime}}=\left.\left(\beta_{2}^{\prime \prime}, \ldots, \beta_{k}^{\prime \prime}\right)\right|_{\text {ker } \beta_{1}^{\prime \prime}}$. For any $X \in$ $L_{k-1}$ with $X^{\prime \prime}=Z$, the tuple $\left(\gamma_{2}^{X^{\prime \prime}}, \ldots, \gamma_{k}^{X^{\prime \prime}}\right)$ consists of elements of $Z_{V(\theta)}^{\perp}$. Furthermore, for any $i, i=2, \ldots, k,\left.\gamma_{i}^{X^{\prime \prime}}\right|_{\operatorname{ker} \beta_{1}^{\prime \prime}}=\left.\beta_{i}^{\prime \prime}\right|_{\operatorname{ker} \beta_{1}^{\prime \prime}}$. Thus by part (ii) of Lemma $5.1, E^{Z}=\left(\gamma_{2}^{X^{\prime \prime}}, \ldots, \gamma_{k}^{X^{\prime \prime}}\right)$. 
We have

$$
\begin{aligned}
\rho\left(\omega_{\mu}\left(\beta_{1}, \ldots, \beta_{k}\right)\right) & =\rho\left(\frac{1}{\beta_{1}} \sum_{X \in L_{k-1}^{Y}} \omega_{\mu_{X}}\left(\gamma_{2}^{X}, \ldots, \gamma_{k}^{X}\right)\right) \\
& =\frac{1}{\beta_{1}^{\prime \prime}} \sum_{X \in L_{k-1}^{Y}} \rho\left(\omega_{\mu_{X}}\left(\gamma_{2}^{X}, \ldots, \gamma_{k}^{X}\right)\right) \\
& =\frac{1}{\beta_{1}^{\prime \prime}} \sum_{X \in L_{k-1}^{Y}} \omega_{h_{k-2}^{X} \mu_{X}}\left(\gamma_{2}^{X \prime \prime}, \ldots, \gamma_{k}^{X \prime \prime}\right) \\
& =\frac{1}{\beta_{1}^{\prime \prime}} \sum_{X \in L_{k-1}^{Y}} \omega_{h_{k-2}^{X} \mu_{X}}\left(E^{X^{\prime \prime}}\right) .
\end{aligned}
$$

By Lemma 6.4, we obtain

$$
\rho\left(\omega_{\mu}\left(\beta_{1}, \ldots, \beta_{k}\right)\right)=\frac{1}{\beta_{1}^{\prime \prime}} \sum_{Z \in L_{k-1}\left(\left(\mathcal{A}_{Y}\right)^{\prime \prime}\right)} \omega_{\diamond}\left(E^{Z}\right) .
$$

Where $\diamond=\sum_{\substack{X \in L_{k-1}^{Y} \\ X^{\prime \prime}=Z}} h_{k-2}^{X} \mu_{X}=\left(h_{k-1}^{Y} \mu\right)_{Z}$. This proves (iv).

To prove that the definition of $\omega_{\mu}(B)$ is, up to scalar multiple, independent of the tuple $B$, we need the following well-known result.

Lemma 6.6. (Chio's Pivotal Condensation Method.) Let $\Delta=\left|\left(\eta_{i j}\right)_{1 \leq i, j \leq k}\right|$ be a determinant. Let $1 \leq m \leq k$.

Let

$$
\Delta^{\prime}=\left|\begin{array}{ccc}
\chi_{12} & \cdots & \chi_{1 k} \\
\cdots & \cdots & \cdots \\
\chi_{m-12} & \cdots & \chi_{m-1 k} \\
\chi_{m+12} & \cdots & \chi_{m+1 k} \\
\cdots & \cdots & \cdots \\
\chi_{k 2} & \cdots & \chi_{k k}
\end{array}\right| \quad \text { where } \quad \chi_{i j}=\left|\begin{array}{cc}
\eta_{m 1} & \eta_{m j} \\
\eta_{i 1} & \eta_{i j}
\end{array}\right|
$$

Then $\Delta^{\prime}=(-1)^{m-1} \eta_{m 1}^{k-2} \Delta$.

Proof. This is an easy generalization of [Ev, Theorem 3.6.1].

The next theorem shows that the definition of $\omega_{\mu}(B)$ is, up to scalar multiple, independent of the tuple $B$.

Theorem 6.7. Let $Y \in L_{k}$, and let $B=\left(\beta_{1}, \ldots, \beta_{k}\right)$ and $E=\left(\eta_{1}, \ldots, \eta_{k}\right)$ be bases of $Y^{\perp}$. Let $\mu \in R_{k}\left(\mathcal{A}_{Y}\right)$. For each $i, 1 \leq i \leq k$, let $\eta_{i}=\sum_{j=1}^{k} \eta_{i j} \beta_{j}$. Let $\Delta$ denote the determinant $\Delta=\left|\left(\eta_{i j}\right)_{1 \leq i, j \leq k}\right|$. Then $\omega_{\mu}(B)=\Delta \omega_{\mu}(E)$. 
Proof. The proof proceeds by induction on $k$.

For $k=2$, we give the argument in case $\eta_{11} \neq 0$. The proof for the only other case, $\eta_{11}=0$ and $\eta_{21} \neq 0$, is similar.

First observe

$$
\eta_{2}=\frac{\eta_{21}}{\eta_{11}} \eta_{1}+\left(\eta_{22}-\frac{\eta_{21} \eta_{12}}{\eta_{11}}\right) \beta_{2}
$$

Now

$$
\begin{aligned}
\omega\left(\beta_{1}, \beta_{2}\right) & =\eta_{11} \omega\left(\eta_{1}, \beta_{2}\right) \\
& =-\eta_{11} \omega\left(\beta_{2}, \eta_{1}\right) \\
& =-\eta_{11}\left(\eta_{22}-\frac{\eta_{21} \eta_{12}}{\eta_{11}}\right) \omega\left(\eta_{2}, \eta_{1}\right) \\
& =\left(\eta_{11} \eta_{22}-\eta_{12} \eta_{21}\right) \omega\left(\eta_{1}, \eta_{2}\right) .
\end{aligned}
$$

This completes the proof for $k=2$.

For $k>2$, assume that the result holds for every $k^{\prime}, 2 \leq k^{\prime}<k$. Choose $m, 1 \leq m \leq k$ so that $\eta_{m 1} \neq 0$. By Theorem 6.5

$$
\begin{aligned}
\omega_{\mu}\left(\beta_{1}, \beta_{2}, \ldots, \beta_{k}\right) & =\eta_{m 1} \omega\left(\eta_{m}, \beta_{2}, \ldots, \beta_{k}\right) \\
& =\frac{\eta_{m 1}}{\eta_{m}} \sum_{X \in L_{k-1}} \omega_{\mu_{X}}\left(\xi_{2}^{X}, \ldots, \xi_{k}^{X}\right) .
\end{aligned}
$$

Where $\left(\xi_{2}^{X}, \ldots, \xi_{k}^{X}\right)$ is a basis of $X^{\perp}$ with $\left.\left(\xi_{2}^{X}, \ldots, \xi_{k}^{X}\right)\right|_{V\left(\eta_{m}\right)}=\left(\beta_{2}, \ldots\right.$, $\left.\beta_{k}\right)\left.\right|_{V\left(\eta_{m}\right)}$.

Note that for $i \neq m$,

$$
\eta_{i}=\frac{\eta_{i 1}}{\eta_{m 1}} \eta_{m}+\sum_{j=2}^{k}\left(\eta_{i j}-\frac{\eta_{i 1}}{\eta_{m 1}} \eta_{m j}\right) \beta_{j} .
$$

Fix an $i, 1 \leq i \leq k$ with $i \neq m$, and consider $\sum_{j=2}^{k}\left(\eta_{i j}-\frac{\eta_{i 1}}{\eta_{m 1}} \eta_{m j}\right) \xi_{j}^{X}$. By (7) and Lemma 5.2, this is a linear functional of the form $\eta_{i}-a \eta_{m}$ for some $a \in \mathbf{K}$. Thus this functional's restriction to $V\left(\eta_{m}\right)$ is exactly the functional $\left.\eta_{i}\right|_{V\left(\eta_{m}\right)}$. It is in $X^{\perp}$, since each $\xi_{j}^{X}$ is in $X^{\perp}$. For $1 \leq i \leq m, i \neq m$, let

$$
\zeta_{i}^{X}=\sum_{j=2}^{k}\left(\eta_{i j}-\frac{\eta_{i 1}}{\eta_{m 1}} \eta_{m j}\right) \xi_{j}^{X} .
$$

Then

$$
\left.\left(\zeta_{1}^{X}, \ldots, \zeta_{m-1}^{X}, \zeta_{m+1}^{X}, \ldots, \zeta_{k}^{X}\right)\right|_{V\left(\eta_{m}\right)}=\left.\left(\eta_{1}, \ldots, \eta_{m-1}, \eta_{m+1}, \ldots, \eta_{k}\right)\right|_{V\left(\eta_{m}\right)}
$$

so, by definition,

$$
\omega_{\mu}\left(\eta_{m}, \eta_{1}, \eta_{2}, \ldots, \hat{\eta}_{m}, \ldots, \eta_{k}\right)=\frac{1}{\eta_{m}} \sum_{X \in L_{k-1}^{Y}} \omega_{\mu_{X}}\left(\zeta_{1}^{X}, \ldots, \hat{\zeta}_{m}^{X}, \ldots, \zeta_{k}^{X}\right) .
$$


By the inductive hypothesis and the definition of $\zeta_{i}^{X}$,

$$
\begin{aligned}
\omega\left(\beta_{1}, \beta_{2}, \ldots, \beta_{k}\right) & =\frac{\eta_{m 1}}{\eta_{m}} \sum_{X \in L_{k-1}^{Y}} \omega_{\mu_{X}}\left(\xi_{2}^{X}, \ldots, \xi_{k}^{X}\right) \\
& =\frac{\eta_{m 1}}{\eta_{m}} \sum_{X \in L_{k-1}^{Y}} \Delta_{1} \omega_{\mu_{X}}\left(\zeta_{1}^{X}, \ldots, \zeta_{m-1}^{X}, \zeta_{m+1}^{X}, \ldots, \zeta_{k}^{X}\right) .
\end{aligned}
$$

Where

$$
\Delta_{1}=\left|\begin{array}{ccc}
A_{12} & \cdots & A_{1 k} \\
\vdots & & \vdots \\
A_{m-12} & \cdots & A_{m-1 k} \\
A_{m+12} & \cdots & A_{m+1 k} \\
\vdots & & \vdots \\
A_{k 2} & \cdots & A_{k k}
\end{array}\right|
$$

and $A_{i j}=\frac{1}{\eta_{m 1}}\left|\begin{array}{cc}\eta_{m 1} & \eta_{m j} \\ \eta_{i 1} & \eta_{i j}\end{array}\right|$

Let

$$
\Delta_{2}=\left|\begin{array}{ccc}
\hat{A}_{12} & \cdots & \hat{A}_{1 k} \\
\vdots & & \vdots \\
\hat{A}_{m-12} & \cdots & \hat{A}_{m-1 k} \\
\hat{A}_{m+12} & \cdots & \hat{A}_{m+1 k} \\
\vdots & & \vdots \\
\hat{A}_{k 2} & \cdots & \hat{A}_{k k}
\end{array}\right|
$$

where $\hat{A}_{i j}=\left|\begin{array}{cc}\eta_{m 1} & \eta_{m j} \\ \eta_{i 1} & \eta_{i j}\end{array}\right|$.

Then by elementary row operations, $\Delta_{1}=\left(\frac{1}{\eta_{m 1}}\right)^{k-1} \Delta_{2}$. Thus

$\omega\left(\beta_{1}, \beta_{2}, \ldots, \beta_{k}\right)=\frac{\left(\frac{1}{\eta_{m 1}}\right)^{k-2} \Delta_{2}}{\eta_{m}} \sum_{X \in L_{k-1}^{Y}} \omega_{\mu_{X}}\left(\zeta_{1}^{X}, \ldots, \zeta_{m-1}^{X}, \zeta_{m+1}^{X}, \ldots, \zeta_{k}^{X}\right)$

By applying Lemma 6.6,

$$
\left(\frac{1}{\eta_{m 1}}\right)^{k-2} \Delta_{2}=\frac{\eta_{m 1}^{k-2}}{\eta_{m 1}^{k-2}}(-1)^{m-1} \Delta=(-1)^{m-1} \Delta
$$


So

$$
\begin{aligned}
\omega\left(\beta_{1}, \beta_{2}, \ldots, \beta_{k}\right) & =\frac{\eta_{m 1}}{\eta_{m}} \sum_{X \in L_{k-1}^{Y}} \Delta_{1} \omega_{\mu_{X}}\left(\zeta_{1}^{X}, \ldots, \zeta_{m-1}^{X}, \zeta_{m+1}^{X}, \ldots, \zeta_{k}^{X}\right) \\
& =(-1)^{m-1} \frac{\Delta}{\eta_{m}} \sum_{Z \in L_{k-1}} \omega_{\mu_{Z}}\left(\zeta_{1}, \ldots, \zeta_{m-1}, \zeta_{m+1}, \ldots, \zeta_{k}\right) \\
& =(-1)^{m-1} \Delta \omega_{\mu}\left(\eta_{m}, \eta_{1}, \ldots, \eta_{m-1}, \eta_{m+1}, \ldots, \eta_{k}\right) \\
& =(-1)^{m-1}(-1)^{m-1} \Delta \omega_{\mu}\left(\eta_{1}, \ldots, \eta_{k}\right) .
\end{aligned}
$$

This proves the theorem.

\section{Type A arrangements.}

Consider a vector space of dimension $l+1$ with basis $u_{1}, \ldots, u_{l+1}$. The Coxeter arrangement of type $A_{l}$ consists of the planes perpendicular to $u_{i}-u_{j}$ for $1 \leq i<j \leq l+1$. This arrangement is the product of an empty 1-arrangement with an irreducible arrangement. We consider here the irreducible factor $\mathcal{A}$ of the Coxeter arrangement of type $A_{l}$. We compute certain elements of the relation spaces associated to $\mathcal{A}$, compute their corresponding logarithmic 1 -forms, and show that they generate the module $\Omega^{1}(\mathcal{A})$. It is an easy consequence that the logarithmic 1-forms described in Section 5 generate all logarithmic 1-forms for Coxeter arrangements of type $A_{l}$. The argument here is field-independent.

For the example in this section, we will need the following well-known property.

Proposition 7.1. (Saito's criterion.) Let $\omega_{1}, \ldots, \omega_{l} \in \Omega_{1}(\mathcal{A})$. Then $\Omega^{1}(\mathcal{A})$ is a free $S$-module with basis $\omega_{1}, \ldots, \omega_{l}$ if and only if $\omega_{1} \wedge \cdots \wedge \omega_{l}$ is a $\mathbf{K}$-multiple of $Q^{-1} d x_{1} \wedge \cdots \wedge d x_{l}$.

Proof. See [OT, Proposition 4.80].

Let $x_{1}, \ldots, x_{l}$ be a basis of $V^{*}$. For $1 \leq p \leq l$, let $X_{p}=V\left(x_{p}\right)$, and for $1 \leq p<q \leq l$, let $Y_{p q}=V\left(x_{p}-x_{q}\right)$. Let $\mathcal{A}$ be the arrangement consisting of all the hyperplanes $X_{p}$ and $Y_{p q}$. We choose linear functionals associated to elements of $\mathcal{A}$ as follows. For $1 \leq p \leq l$, let $\alpha_{X_{p}}=x_{p}$, and for $1 \leq p<q \leq l$, let $\alpha_{Y_{p q}}=x_{p}-x_{q}$.

For $1 \leq p \leq l$, denote the basis element of $\bigoplus_{X \in L_{1}} R_{1}\left(\mathcal{A}_{X}\right)$ corresponding to $X_{p}$ by $e_{p}$, and for $1 \leq p<q \leq l$, denote the basis element corresponding to $Y_{p q}$ by $e_{p q}$.

We now recursively compute certain elements of the spaces $R_{k}\left(\mathcal{A}_{X}\right)$ for $k \geq 2$. For $k \geq 2$ and $1 \leq i_{1}<i_{2}<\cdots<i_{k} \leq l$, let $X_{i_{1} \cdots i_{k}}=$ $V\left(x_{i_{1}}, \ldots, x_{i_{k}}\right) \in L_{k}$, and let $Y_{i_{1} \cdots i_{k}}=V\left(x_{i_{1}}-x_{i_{2}}, x_{i_{1}}-x_{i_{3}}, \ldots, x_{i_{1}}-x_{i_{k}}\right) \in$ $L_{k-1}$. Observe that this is consistent with the definition of $Y_{p q}$ above. 
For every $p$ and $q$ with $1 \leq p<q \leq l$ we have a relation $\mu_{p q}=e_{p}-e_{q}-$ $e_{p q} \in R_{2}\left(\mathcal{A}_{X_{p q}}\right)$, and for every $p, q$, and $r$ with $1 \leq p<q<r \leq l$, we have a relation $\nu_{p q r}=-e_{p q}+e_{p r}-e_{q r} \in R_{2}\left(\mathcal{A}_{Y_{p q r}}\right)$.

These relations generalize recursively as follows. For $k \geq 3$ and $1 \leq i_{1}<$ $\cdots<i_{k} \leq l$, let

$$
\mu_{i_{1} \cdots i_{k}}=\sum_{j=1}^{k}(-1)^{j} \mu_{i_{1} \cdots \hat{i}_{j} \cdots i_{k}}+\nu_{i_{1} \cdots i_{k}} \in R_{k}\left(\mathcal{A}_{X_{i_{1} \cdots i_{k}}}\right),
$$

and let

$$
\nu_{i_{1} \cdots i_{k}}=\sum_{j=1}^{k}(-1)^{j+1} \nu_{i_{1} \cdots \hat{i}_{j} \cdots i_{k}} \in R_{k-1}\left(\mathcal{A}_{Y_{i_{1} \cdots i_{k}}}\right) .
$$

It now follows by direct computation and recursion that, for any $k \geq 2$ and $1 \leq i_{1}<\cdots<i_{k} \leq l$, we have

$\omega_{\mu_{i_{1} \cdots i_{k}}}\left(x_{i_{1}}, \ldots, x_{i_{k}}\right)=\sum_{j=1}^{k}(-1)^{j+1} \frac{d x_{i_{j}}}{x_{i_{j}} \prod_{1 \leq p<j}\left(x_{i_{p}}-x_{i_{j}}\right) \prod_{j<p \leq k}\left(x_{i_{j}}-x_{i_{p}}\right)}$.

Proposition 7.1 now implies that the forms

$$
\omega_{i_{1}}\left(x_{1}\right), \omega_{i_{1} i_{2}}\left(x_{1}, x_{2}\right), \ldots, \omega_{i_{1} i_{2} \cdots i_{l}}\left(x_{1}, x_{2}, \ldots, x_{l}\right)
$$

constitute a basis for the free $S$-module $\Omega^{1}(\mathcal{A})$.

Acknowledgements. The author would like to extend thanks to Sergey Yuzvinsky for many valuable comments and suggestions made during the completion of this paper. This manuscript was prepared using Paul Taylor's macro file entitled "Tex macros for drawing category-theoretic diagrams".

\section{References}

[Br] K. Brandt, A combinatorial study of the module of derivations of an arrangement of hyperplanes, Ph. D. Thesis, University of Wisconsin-Madison, 1992.

[BT] K. Brandt and H. Terao, Free arrangements and relation spaces, Disc. Comput. Geom., 12 (1994), 49-63.

[De] P. Deligne, Théorie de Hodge, Publ. Math. IHES, 40 (1972), 5-57.

[Ev] H. Eves, Elementary matrix theory, Dover, New York, NY, 1980.

[JS] T. Jósefiak and B. Sagan, Basic derivations for subarrangements of Coxeter arrangements, J. Alg. Comb., 2 (1993), 291-320.

[OT] P. Orlik and H. Terao, Arrangements of hyperplanes, Grundleheren 300, SpringerVerlag, New York, NY, 1992.

[RT] L. Rose and H. Terao, A free resolution of the module of logarithmic forms of a generic arrangement, J. of Algebra, 136 (1991), 376-400. 
[Sa] K. Saito, Theory of logarithmic differential forms and logarithmic vector fields, J. Fac. Sci. Univ. Tokyo, Sect. IA, 27 (1980), 265-291.

$[\mathrm{Yu}] \mathrm{S}$. Yuzvinsky, On generators of the module of logarithmic 1-forms with poles along an arrangement, J. Alg. Comb., 4 (1995), 253-269.

[Za] T. Zaslavsky, Facing up to arrangements: Face-count formulas for partitions of space by hyperplanes, Memoirs Amer. Math. Soc., 154 (1975).

[Zi] G.M. Ziegler, Algebraic combinatorics of hyperplane arrangements, Adv. in Math., 76 (1989), 116-154.

Received July 28, 1997 and revised October 7, 1997.

Department of Mathematics and Computer Science

Grinnell College

GRINNELL, IA 50112

E-mail address: simig@blue.weeg.uiowa.edu 